\title{
A Case Report of Refractory Gout: Efficacy of High-Dose Anti-IL1r Treatment
}

\author{
Leila A. Cardoso ${ }^{\text {a, e }}$, Joao Pedro Abreu ${ }^{\text {a, b }}$, Yenny Anzola ${ }^{a, c}$, Susan Foreid ${ }^{\text {, }}$ \\ Isabel Almeida ${ }^{\text {a }}$, Antonio Marinho ${ }^{\mathrm{a}}$, Fatima Farinha ${ }^{\mathrm{a}}$
}

\begin{abstract}
Gout is an inflammatory disease characterized by the deposition of monosodium urate (MSU) crystals in joints and other tissues. In a significant portion of patients, hyperuricemia is part of the metabolic syndrome; therefore, secondary causes of gout should be looked for and treated. The authors present a case of a persistent and refractory gout, despite standard therapeutics, leading to the need to explore a new pharmacological approach - anti-IL1r.
\end{abstract}

Keywords: Gout; Monosodium urate Crystals; Metabolic syndrome; Anakinra

\section{Introduction}

Gout is an inflammatory disease characterized by the deposition of monosodium urate (MSU) crystals in joints and other tissues. The formation of the crystals is the consequence of hyperuricemia, a condition so called when serum uric acid (SUA) levels are $>6.0 \mathrm{mg} / \mathrm{dL}(360 \mu \mathrm{mol} / \mathrm{L})$ [1]. The MSU crystals are one of the most powerful inflammatory triggers in men. The sequence of events following MSU precipitation concerns their physical contact with cells and the interaction with both surface and intracellular receptors that finally leads to the activation of specific inflammatory pathways. Their presence leads to monocytes and macrophages activation, with the production of chemotactic substances causing a massive neutrophil infiltration in joint fluid and synovial membrane, inducing the secretion of cytokines, prostanoids, chemotactic

Manuscript accepted for publication March 17, 2017

aUnidade de Imunologia Clinica do Porto, Centro Hospitalar do Porto, Porto, Portugal

bServico de Medicina Interna do Hospital Santa Maria Maior, Barcelos, Portugal

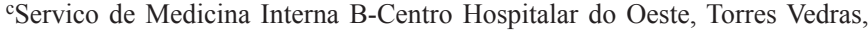
Portugal

dAnatomia Patologica, Laboratorio Dr Macedo Dias - SYNLAB Group, Porto, Portugal

${ }^{\mathrm{e} C o r r e s p o n d i n g ~ A u t h o r: ~ L e i l a ~ A m a r o ~ C a r d o s o, ~ S e r v i c o ~ d e ~ M e d i c i n a ~ I n t e r n a ~}$ B, Hospital Geral do Centro Hospitalar Universitario de Coimbra, 3046-853 Coimbra, Portugal. Email: leilaamarocardoso@gmail.com

doi: https://doi.org/10.14740/jmc2790w factors and other proteins. This mechanism amplifies the inflammatory process through the recruitment of inflammatory cells, the upregulation of adhesion molecules and the stimulation of acute-phase response [2]. In Western countries, gout affects about $1-2 \%$ of adults, with a prevalence increasing with age, being $7 \%$ in men over 65 years and $3 \%$ in women over 85 years [3]. In a significant portion of patients, hyperuricemia is part of the metabolic syndrome; therefore, secondary causes of gout should be looked for and treated [4]. Identification of MSU crystals in synovial fluid (SF) obtained during the attacks [5], from previously inflamed asymptomatic joints of untreated subjects during intercritical periods [6] or in an aspirate from a tophus is simple and allows immediate unequivocal diagnosis.

The management of gout may include different approaches depending on the type of disease presentation and individual patient characteristics [1]. However, the main aims of treatment are the treatment of acute attacks and the cure and/or the prevention of chronic disease, by achieving dissolution of the pathogenic MSU crystals.

The authors present a case of a persistent and refractory gout attack, focusing on the therapeutic approach.

\section{Case Report}

We present a 49-year-old male patient, with morbid obesity (body mass index $>35$ ), on onset with severe and incapacitating polyarthritis of small and large joints, with 1 month of evolution. Evaluation showed asymmetric nodular arthritis, compatible with gout tophus. Serum workout showed hyperuricemia $(13.4 \mathrm{mg} / \mathrm{dL})$. Colchicine $(1 \mathrm{mg} /$ day $)$ was started on the first appointment for acute gout management and prophylaxis. Two months later, patient showed worsening of the symptoms, with the same polyarthritis pattern, with ongoing treatment. Since the lack of therapeutics response and the possible overlap with rheumatoid arthritis (RA), corticotherapy (deflazacort $15 \mathrm{mg}$ per day) and allopurinol were started with colchicine interruption. One month later, patient showed great response to corticotherapy, not only a clinical one, but also an analytic one (with reduction of uric acid levels on serum, despite still over $10 \mathrm{mg} / \mathrm{dL})$.

At that moment, blood workout showed significant inflammatory markers elevation, dyslipidemia (majorly hypertriglyceridemia), rheumatoid factor borderline and negative $\mathrm{Ab}$ anti-CCP. Secondary causes of gout were accessed and addressed concomitantly. 


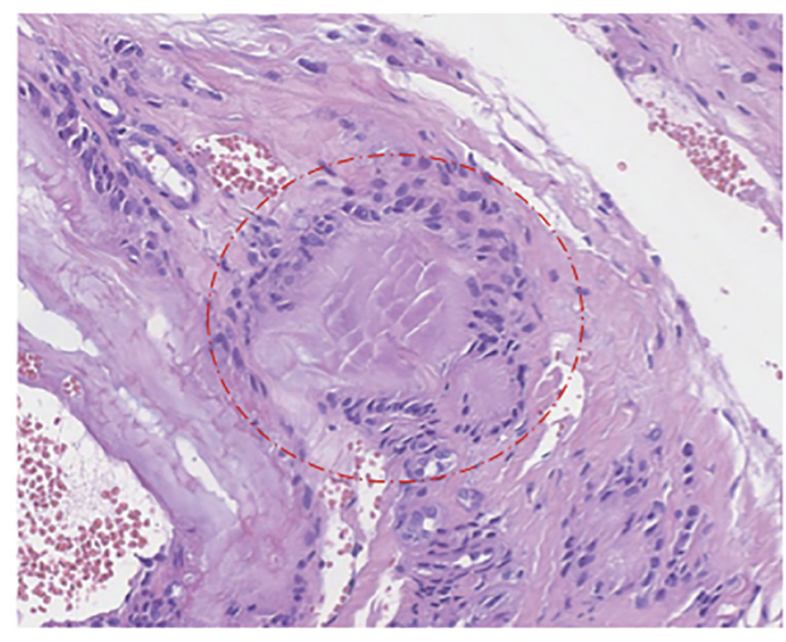

Figure 1. Typical granuloma in gout. The central part is formed by urate crystals. The inflammatory cells surrounding the area of crystals typical include macrophages, lymphocytes, plasma cells and giant cells. This is typical morphology within tophi. No histologic morphology of a coexisting rheumatoid arthritis was present. Fixation: formalin. H\&E: amplification, $\times 20$.

Despite therapeutics and symptoms amelioration, no remission was achieved, with the patient maintaining disease activity.

Throughout corticotherapy progressive withdrawal, patient showed worsening of the symptoms (deflazacort $6 \mathrm{mg}$ / day), at the moment with peripheral arthritis (wrists and tibiotarsus). Although without positivity to anti-citrullinated antibodies, and rheumatoid factor borderline, patient initiated methotrexate (MTX) orally (15 mg/week) assuming the possibility of a gout overlap with seronegative rheumatoid arthritis. Patient showed good response with MTX and corticoid, during 2 months, and then had adverse reaction to MTX, with severe herpetic stomatitis, thrombocytopenia, and mild hepatitis. MTX was suspended, and high-dose corticotherapy was initiated - prednisolone $1 \mathrm{mg} / \mathrm{kg} /$ day. Patient showed good response to therapeutics decisions, yet inability to tolerate less than $20 \mathrm{mg}$ of prednisolone per day, due to arthritis flares.

In summary, at that moment, we had an obese patient, with a metabolic syndrome and a severe form of gout, unresponsive to colchicine and low doses of corticoid. This is a highly active disease, with persistently high uric acid levels. Therefore, we decided a histological approach to one of the nodular lesions, revealing granulomas with deposition of MSU crystals, as shown in Figure 1. It is concluded a severe form of gout, with continuous polyarthritis, unresponsive to standard treatment, excluding the possibility of an RA overlap.

Since the disease severity and the persistently high uric acid levels, despite allopurinol (during periods of remission), the case was presented in a multidisciplinary committee, being decided to start anti-IL1r antagonist (Anakinra ${ }^{\circledR}$ ) $100 \mathrm{mg} /$ day and rasburicase, maintaining corticotherapy in the lower dose tolerated (20 mg/day).

With treatment, there was reduction of the inflammatory response, with tolerance to low-dose corticoid, although maintaining moderate outbreaks. Rasburicase was mildly effective on diminishing uric acid serum levels, yet not to normal.

Due to recurrent milder outbreaks, we gradually increased Anakinra ${ }^{\circledR}$ dosage to $200 \mathrm{mg}$ /day, adjusted to patient's weight. With this measure, total remission was accomplished.

As we consider obesity as a hallmark to the refractory aspect of this gout case, at the moment stable with Anakinra ${ }^{\circledR}$, low-dose corticotherapy and rasburicase, the patient is now waiting for bariatric surgery.

\section{Discussion}

Although clinical manifestations may be typical, and mostly sufficient to the diagnosis, cases of refractory symptoms to standard therapeutics, or atypical clinical presentations may require different and more invasive diagnostic strategies, such as the intraarticular SF sampling [7]. In fact, the last can be useful and even mandatory in the diagnosis of gout or when there is a suspicion of gout [7]. The identification of MSU crystals in SF samples drawn from joints undergoing gouty attacks or from tophi allows an unequivocal definitive diagnosis of gout [8].

In our case, the difficulty of the diagnosis lied in the absence of remission, even the intense disease activity, throughout the time in which the patient was undergoing standard gout treatment. Due to the symptoms severity, different pathologies were admitted, which may have delayed the definitive diagnosis, considering overlapping diseases, such as RA as mentioned before. The SF sample was mandatory to clarify the case, having then confirmed gout.

The most appropriate drugs for gout treatment are antiinflammatory substances, including traditional drugs such as colchicine, non-steroidal anti-inflammatory drugs (NSAIDs) and steroids, and innovative drugs, such as the biological drugs acting as IL-1 inhibitors, in patients with inadequate response or contraindication/intolerance to standard drugs [9].

Our patient, as we presented, had been treated, during acute outbreaks, with all the drugs already announced, except biological alternatives, with poor response. Only high dosage of corticoid was mildly effective. Therefore, IL-1 blockers were an alternative to be considered. With the initial dose of $100 \mathrm{mg} /$ day, a moderate response was achieved, despite persistently high levels of uric acid.

As we know, patients with hyperuricemia and/or gout should be carefully assessed for the presence of metabolic syndrome, since its prevalence may be very high, up to $60-70 \%$, and is a well-known contributor to refractory hyperuricemia [4]. Our subject was poorly responsive to drugs that reduce uric acid levels, which can partially explain the refractory symptomatology, and the necessity of high intensity treatments. Having this fact in account, the patient was proposed to bariatric surgery. While in waiting list, the IL-1 blocker (Anakinra) was gradually titrate to $200 \mathrm{mg}$ /day with better clinical control, without any adverse effects, until the moment.

\section{Conclusion}

We present a severe gout, refractory to the standard treatment, 
in a metabolic syndrome patient, in which the need to explore different therapeutics defined our approach.

The IL-1 blockers are FDA and European Medicines Agency (EMA) approved agents for rheumatoid arthritis. These drugs have recently been investigated in refractory gout, with promising results in both acute attack and prophylaxis.

This patient, despite all the therapeutic strategies to disease activity control, including non-pharmacological measures and standard drugs, remained severely impaired to perform daily activities. The IL-1 blockers, in this case, were important to reduce symptoms and improve life quality.

Despite being rather effective in providing significant pain and inflammation relief and reduction of the risk of new flares in patients with acute gouty arthritis, clinicians must not forget to access risk factors, as the metabolic syndrome, and address them in both pharmacological and non-pharmacological way.

No doubt the benefits of appropriate lifestyle changes go far beyond their effect on gout.

In selected patients, continuous anakinra treatment, even dose titrating, may be considered as an alternative approach to avoid high steroid dosage and address refractory symptoms.

\section{References}

1. Bardin T, Richette P. Definition of hyperuricemia and gouty conditions. Curr Opin Rheumatol. 2014;26(2):186191.
2. Choi HK, Mount DB, Reginato AM. Pathogenesis of gout. Ann Intern Med. 2005;143(7):499-516.

3. Roddy E, Doherty M. Epidemiology of gout. Arthritis Res Ther. 2010;12(6):223.

4. Pascual E, Sivera F. Time required for disappearance of urate crystals from synovial fluid after successful hypouricaemic treatment relates to the duration of gout. Ann Rheum Dis. 2007;66(8):1056-1058.

5. McCarty DJ, Hollander JL. Identification of urate crystals in gouty synovial fluid. Ann Intern Med. 1961;54:452460.

6. Pascual E, Batlle-Gualda E, Martinez A, Rosas J, Vela P. Synovial fluid analysis for diagnosis of intercritical gout. Ann Intern Med. 1999;131(10):756-759.

7. Punzi L, Oliviero F. Arthrocentesis and synovial fluid analysis in clinical practice: value of sonography in difficult cases. Ann N Y Acad Sci. 2009;1154:152-158.

8. Zhang W, Doherty M, Pascual E, Bardin T, Barskova V, Conaghan P, Gerster J, et al. EULAR evidence based recommendations for gout. Part I: Diagnosis. Report of a task force of the Standing Committee for International Clinical Studies Including Therapeutics (ESCISIT). Ann Rheum Dis. 2006;65(10):1301-1311.

9. Zhang W, Doherty M, Bardin T, Pascual E, Barskova V, Conaghan P, Gerster J, et al. EULAR evidence based recommendations for gout. Part II: Management. Report of a task force of the EULAR Standing Committee for International Clinical Studies Including Therapeutics (ESCISIT). Ann Rheum Dis. 2006;65(10):1312-1324. 